\title{
Microlensing Constraints on Low-Mass Companions
}

\author{
B. Scott Gaudi \\ Hubble Fellow, School of Natural Sciences, Institute for Advanced Study, \\ Einstein Drive, Princeton, NJ, 08540
}

\begin{abstract}
Microlensing is sensitive to binary, brown dwarf (BD), and planetary companions to normal stars in the Galactic bulge with separations between about 1-10 AU. The accurate, densely-sampled photometry of microlensing events needed to detect planetary companions has been achieved by several follow-up collaborations. Detailed analysis of microlensing events toward the bulge demonstrates that less than $45 \%$ of M-dwarfs in the bulge have $M_{\text {Jup }}$ companions between 1 and 5 AU. Detection of binary and BD companions using microlensing is considerably easier; however, the interpretation is hampered by their non-perturbative influence on the parent lightcurve. I demonstrate that $\sim 25 \%$ of $\mathrm{BD}$ companions with separations $1-10 \mathrm{AU}$ should be detectable with surveyquality data $(\sim 1$ day sampling and $\sim 5 \%$ photometry). Survey data is more amenable to generic, brute-force analysis methods and less prone to selection biases. An analysis of the $\sim 1500$ microlensing events detected by OGLE-III in the next three years should test whether the BD desert exists at separations $1-10 \mathrm{AU}$ from $\mathrm{M}$-dwarfs in the Galactic bulge.
\end{abstract}

\section{Introduction}

It is by now well-established by radial velocity (RV) surveys that the mass function of low-mass companions to normal (GKM) stars in the solar neighborhood exhibits a "brown-dwarf (BD) desert," a paucity of $13-80 M_{\text {Jup }}$ companions. Specifically, $<1 \%$ of solar-type stars have BD companions with semi-major axes $a \lesssim 5 \mathrm{AU}$ (Marcy \& Butler 2000). Although RV surveys will eventually be able to detect more distant companions, the duration of their observations are currently only sufficient to detect long-term trends from companions with $a \gtrsim 5 \mathrm{AU}$ (See Figure 1). Young BD companions with separations greater than a few tens of AUs and less than a few hundreds of AUs can be detected via direct imaging surveys (Oppenheimer et al. 2001, Lowrance 2001, McCarthy 2001); even more distant companions are detectable serendipitously in wide-field surveys (Gizis et al. 2001). These studies seem to suggest that the frequency of wide BD companions to GKM stars is $\lesssim 10 \%$ (see McCarthy 2001). Neither RV nor direct imaging can currently assess the frequency of companions with $a=5-10 \mathrm{AU}$. The exquisite precision $\left(\sim 3 \mathrm{~km} \mathrm{~s}^{-1}\right)$ of RV surveys also makes them sensitive to planetary companions with mass $0.1-13 M_{\mathrm{Jup}}$; currently nearly 100 such companions are now known. The $M \sin i$ and $a$ of these companions are shown in Figure 1. There also appears to be a statistically significant dearth of high-mass 
$\left(M>5 M_{\mathrm{Jup}}\right)$, close-in $(a \lesssim 0.3 \mathrm{AU})$ planetary companions (Zucker \& Mazeh 2002; see also Figure 1). Whether this is related to the BD desert is not clear.

Microlensing is sensitive to binary, $\mathrm{BD}$, and planetary companions separated by $\sim 1-10 \mathrm{AU}$ from the objects in the Galactic bulge which serve as the lenses of microlensing events detected toward the Galactic bulge (Mao \& Paczyński 1991). The majority of the primaries are likely to be M-dwarfs in the bulge, with some contamination from stellar remnants and disk stars. Microlensing is sensitive to the mass ratio $q$ and projected separation $d$ of binary systems, where $d$ is in units of the combined Einstein ring radius $r_{\mathrm{E}}$ of the system. I will assume that the primaries are M-dwarfs with $M=0.3 M_{\odot}$ in the bulge, so that $r_{\mathrm{E}}=2 \mathrm{AU}$, and use these to transform to the mass $M_{p}$ and semi-major axis $a$ of the secondaries.

\section{Microlensing Constraints on Planetary Companions}

The collaborations that survey the Galactic bulge generally have insufficient sampling ( $\sim$ day) to detect the deviations caused by planetary companions to the primary lenses, which last for a fraction $\sim \sqrt{q}$ of the total event duration, or $\sim 1$ day $\left(M_{p} / M_{\mathrm{Jup}}\right)^{1 / 2}$ for typical parameters. However, these surveys reduce their data real-time, allowing them to issue alerts of ongoing events. Follow-up collaborations can then monitor only promising events and optimize exposure times and sampling on an event-by-event basis. Several such collaborations have demonstrated that the precision and sampling needed to detect Jupiter-mass companions is readily achievable (Rhie et al. 2000; Albrow et al. 2001; Tsapras et al. 2001, Bond et al. 2002).

Analyzing events for the presence of planetary signals is made considerably easier by the perturbative nature of the planetary signal for $q \lesssim 0.01$ : such events are well-described by short-duration deviations atop otherwise normal (singlelens) lightcurves. Therefore, selecting the sample of events suitable for analysis is straightforward, and searching for planetary signals and determining detection efficiencies is also considerably less complicated and time-consuming. A detailed analysis of 43 events monitored by the PLANET collaboration uncovered no candidate planetary signals. This null result was used to place an upper limit to the fraction of lenses that hosted companions. Figure 1 shows the region of parameter space in which $<45 \%$ of M-dwarfs in the bulge can have companions at $95 \%$ confidence (Albrow et al. 2001, Gaudi et al. 2002).

\section{Microlensing Constraints on Brown Dwarf Companions}

For $q \gtrsim 0.01$, the perturbative nature of the companion begins to break down, and the resulting lightcurves begin to exhibit considerably more complexity. This introduces several complications to the analysis. First, the relation between the lightcurve parameters and the observable features is no longer straightforward. This means that it is difficult to estimate parameters without detailed fitting; however detailed fitting is generally quite difficult because of the highly pathological nature of the $\chi^{2}$ surface. These difficulties are exacerbated in densely-sampled, high-quality lightcurves, which tend to sample high- 


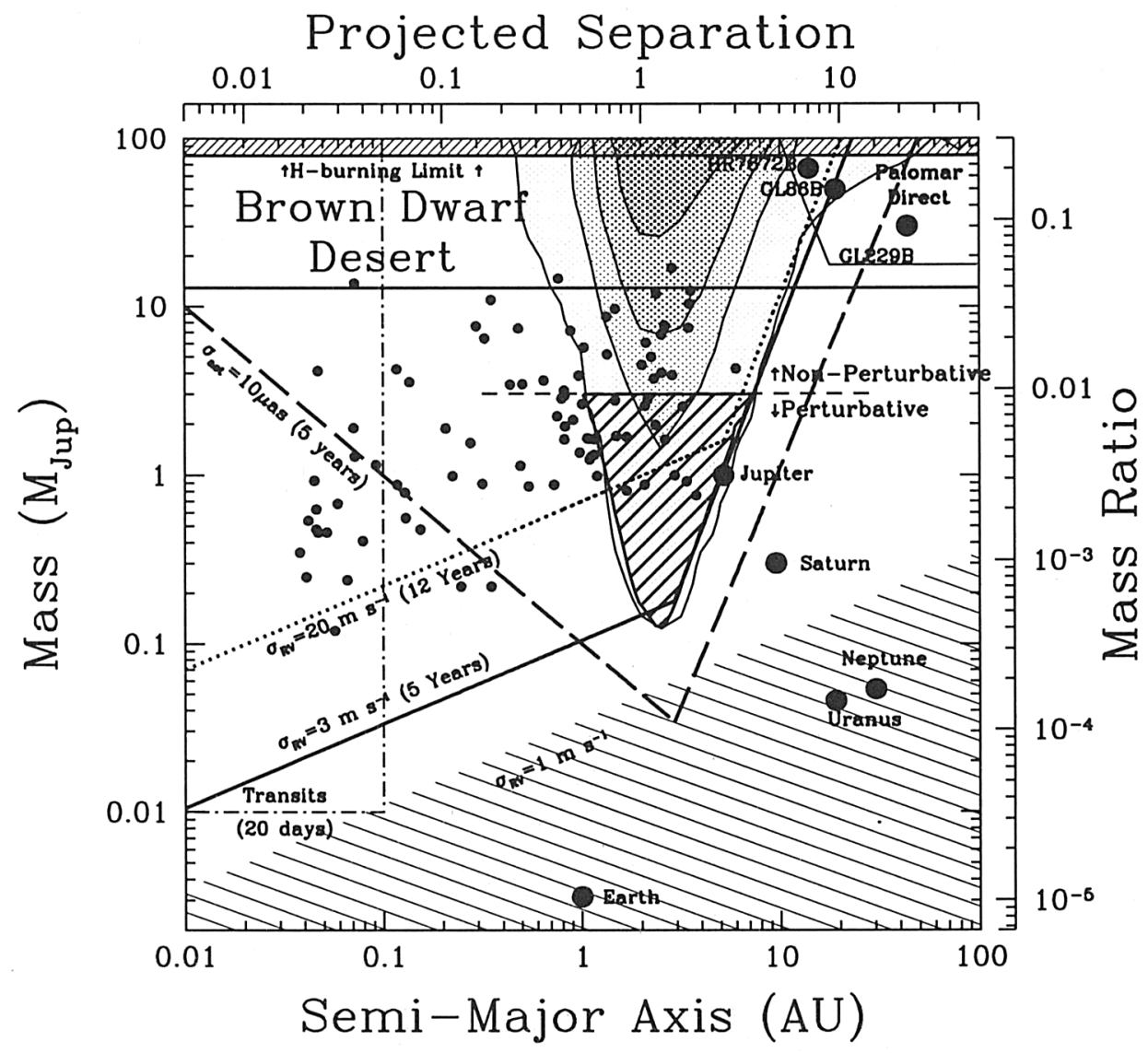

Figure 1. Census of brown dwarf and planetary companions to solartype stars, as well as sensitivities of various detection methods. The small points are companions detected in radial velocity (RV) surveys; the detection limits of such surveys are shown for the durations and precisions indicated, assuming solar-mass primaries. The hatched region in the lower right is inaccessible to RV surveys. The detection limit for an astrometric survey of solar-mass primaries at $10 \mathrm{pc}$ is shown as the long-dashed line. The dot-dashed line shows the approximate limit for a 20 day transit survey. The exclusion region from the PLANET microlensing survey is shown as the triangular hatched region; $<45 \%$ of M-dwarfs in the bulge have companions in this region. The region of sensitivity of the Palomar search is shown as the quadrilateral in the top right corner. The contours show microlensing detection probabilities of $2.5 \%, 25 \%, 50 \%, 75 \%$ (outer to inner) assuming daily sampling and $5 \%$ photometry. 
magnification portions of the lightcurve, whose precise shape depend sensitively on minute variations in the underlying parameters. Furthermore, since a large portion of such lightcurves will appear anomalous, they will generally be recognized early on, and thus preferentially followed by the follow-up collaborations. This introduces hard-to-quantify selection biases. Therefore, it is likely to be considerably easier to study $q \gtrsim 0.01$ companions with survey photometry, since it will be more amenable to global brute-force analysis methods and less-prone to selection biases (Jaroszyński 2002).

The number of events that must be analyzed to give interesting constraints on $\mathrm{BD}$ companions depends on the sensitivity of survey-quality photometry. I estimate this by determining the detection efficiency as a function of $\left(M_{p}, a\right)$, assuming uniform daily sampling between 60 days before until 60 days after the peak, an event timescale of 20 days, a photometric accuracy of $5 \%$, and a uniform distribution of impact parameters up to 0.8 . The resulting contours of constant detection efficiency are shown in Figure 1. I find that $\sim 25 \%$ of $\mathrm{BD}\left(M_{p}=13-80 M_{\mathrm{Jup}}\right)$ companions with separations $1-10 \mathrm{AU}$ are detectable with survey photometry. Therefore, in $N$ microlensing events, the number of BD detections is approximately $N_{\mathrm{BD}}=0.2 f_{\mathrm{BD}} N$, where $f_{\mathrm{BD}}$ is the frequency of BDs with $a=1-10 \mathrm{AU}$. OGLE-III (http://www.astrouw.edu.pl/ ftp/ogle/) should detect $\sim 500$ events per year (Udalski et al. 2002); therefore fractions of $f_{\mathrm{BD}} \lesssim 1 \%$ can be probed in the next few years.

Acknowledgments. Support for this work was provided by NASA through a Hubble Fellowship grant from the Space Telescope Science Institute, which is operated by the Association of Universities for Research in Astronomy, Inc., under NASA contract NAS5-26555.

\section{References}

Albrow, M. D. et al. 2001, ApJ, 556, L113

Bond, I. A. et al. 2002, MNRAS, 333, 71

Gaudi, B. S. et al. 2002, ApJ, 566, 463

Gizis, J.E., Kirkpatrick, J.D., Burgasser, A., Reid, I.N., Monet, D.G., Liebert, J., Wilson, J.C. 2001, ApJ, 551, L163

Jaroszyński, M. 2002, Acta Astron., accepted (astro-ph/0203476)

Lowrance, P.J. 2001, Ph.D. Thesis, UCLA

Mao, S., \& Paczyński, B. 1991, ApJ, 374, 37

Marcy, G. W. \& Butler, R. P. 2000, PASP, 112, 137

McCarthy, C. 2001, Ph.D. Thesis, UCLA

Oppenheimer, B. R., Golimowski, D. A., Kulkarni, S. R., Matthews, K., Nakajima, T., Creech-Eakman, M., \& Durrance, S. T. 2001, AJ, 121, 2189

Rhie, S. H. et al. 2000, ApJ, 533, 378

Tsapras, Y. et al. 2001, MNRAS, 325, 1205

Udalski, A. et al. 2002, Acta Astronomica, 52, 1

Zucker, S. \& Mazeh, T. 2002, ApJ, 568, L113 